\title{
Wide-field multiphoton imaging with TRAFIX
}

Adrià Escobet-Montalbán, Philip Wijesinghe, Mingzhou Chen, Kishan Dholakia

Adrià Escobet-Montalbán, Philip Wijesinghe, Mingzhou Chen, Kishan Dholakia, "Wide-field multiphoton imaging with TRAFIX," Proc. SPIE 10882, Multiphoton Microscopy in the Biomedical Sciences XIX, 108821G (22 February 2019); doi: 10.1117/12.2508373

SPIE. Event: SPIE BiOS, 2019, San Francisco, California, United States 


\title{
Wide-field multiphoton imaging with TRAFIX
}

\author{
Adrià Escobet-Montalbán ${ }^{\mathrm{a}}$, Philip Wijesinghe ${ }^{\mathrm{a}}$, Mingzhou Chen ${ }^{\mathrm{a}}$, and Kishan Dholakia ${ }^{\mathrm{a}}$ \\ ${ }^{a}$ SUPA, School of Physics and Astronomy, University of St Andrews, North Haugh, St \\ Andrews, KY16 9SS, UK.
}

\begin{abstract}
Optical approaches have broadened their impact in recent years with innovations in both wide-field and superresolution imaging, which now underpin biological and medical sciences. Whilst these advances have been remarkable, to date, the ongoing challenge in optical imaging is to penetrate deeper. TRAFIX is an innovative approach that combines temporal focusing illumination with single-pixel detection to obtain wide-field multiphoton images of fluorescent microscopic samples deep through scattering media without correction. It has been shown that it can image through biological samples such as rat brain or human colon tissue up to a depth of seven scattering mean-free-path lengths. Comparisons of TRAFIX with standard point-scanning two-photon microscopy show that the former can yield a five-fold higher signal-to-background ratio while significantly reducing photobleaching of the specimen. Here, we show the first preliminary demonstration of TRAFIX with three-photon excitation imaging dielectric beads. We discuss the advantages of the TRAFIX approach combined with compressive sensing for biomedicine.
\end{abstract}

Keywords: temporal focusing, single-pixel imaging, scattering media, multiphoton microscopy, three-photon, compressive sensing.

\section{INTRODUCTION}

Fluorescence microscopy has become the method of choice for many imaging studies in the biomedical sciences. The capability of super-resolution has enabled the imaging of minute details within cells that were invisible to standard optical devices. ${ }^{1-3}$ Fast volumetric wide-field techniques, such as light-sheet fluorescence microscopy, have made it possible to image whole organisms in a fast and minimally invasive capacity. ${ }^{4}$ These microscopes work extraordinarily well with relatively small and transparent samples; however, in the presence of turbidity, i.e. in opaque biological samples, light is strongly scattered and imaging performance is compromised.

Multiple approaches have recently aimed to overcome this challenge, but the optimal technique for deep imaging is yet to be established. Adaptive optics, initially developed for astronomy, can significantly improve image quality. ${ }^{5}$ However, the amount of correction that deformable mirrors can introduce is insufficient to overcome the effects of scattering in many challenging conditions. A strong effort is currently being made in the characterization of complex media ${ }^{6}$ and focusing through turbidity. ${ }^{7}$ The effects of scattering media on a laser beam can now be characterized and compensated for. In this way, for instance, scattering materials can be used as lenses to focus light into a spot. Although this research field is truly remarkable, the imaging of twoor three-dimensional samples is not currently practical since correction has to be performed in a point-by-point manner, making the technique complex and time-consuming.

The nonlinear two-photon (2P) excitation process has been applied to point-scanning microscopy. Conventionally, a tightly focused beam is scanned across the sample pixel by pixel. ${ }^{8,9}$ The long illumination wavelength enables the excitation of deeper structures within the sample. The generated fluorescence intensity is measured with a fast photo-detector, such as a photomultiplier tube (PMT). In this way, no spatial information needs to be resolved by the detection system and deeper imaging is achieved. Intriguingly, it has been shown that focusing an ultrashort pulse in time may be more appropriate for deep imaging through scattering than traditional spatial focusing since the temporal and the spatial domains are affected differently by scattering. ${ }^{10}$ Exploiting this

Further author information: (Send correspondence to K.D.)

A.E-.M.: E-mail: aem23@st-andrews.ac.uk, Telephone: +44 (0)1334 463124

K.D.: E-mail: kd1@st-andrews.ac.uk, Telephone: +44 (0)1334 463184

Multiphoton Microscopy in the Biomedical Sciences XIX, edited by Ammasi Periasamy, Peter T. C. So, Karsten König, Proc. of SPIE Vol. 10882, 108821G · @ 2019 SPIE · CCC code: 1605-7422/19/\$18 · doi: 10.1117/12.2508373

Proc. of SPIE Vol. 10882 108821G-1 
property, temporal focusing has emerged as a new way to achieve fast wide-field multiphoton imaging, ${ }^{11-13}$ as well as multiplexed excitation for optogenetics ${ }^{14-16}$ in scattering media. Recently, multiphoton imaging has been extended to the three-photon (3P) excitation regime. Its use in point-scanning, ${ }^{17-21}$ light-sheet ${ }^{22}$ and temporal focusing ${ }^{23}$ schemes has reached unprecedented imaging depths in biological samples.

Separately, single-pixel cameras have been employed to take images in challenging situations, in which standard pixel-array cameras may not offer the optimal approach. ${ }^{24,25}$ In this scheme, a digital micromirror device (DMD) or a liquid crystal spatial light modulator (SLM) spatially patterns the illumination field or detection field. Light intensity reflected or emitted by the sample is then recorded with a single-element detector, such as a photomultiplier tube (PMT), and computational imaging methods are used to retrieve spatial information from the sample.

In our recently developed technique, termed TRAFIX, we have combined patterned temporal focusing illumination with single-pixel detection to achieve deep wide-field multiphoton imaging without correction in scattering samples. ${ }^{26}$ We showed that it competes favorably with standard two-photon point-scanning microscopy, showing a five-fold improvement in signal-to-background ratio and reduced photobleaching. In this paper, we use $2 \mathrm{P}$ excitation TRAFIX images of fluorescent beads embedded in a scattering phantom to demonstrate that the use of compressive sensing can conserve image fidelity whilst substantially reducing the volume and speed of the acquisition. ${ }^{24,25,27}$ We also show that the microscope can be used in $3 \mathrm{P}$ excitation mode by showing a preliminary investigation where we image fluorescent microspheres, in this case without the presence of any scattering medium.

\section{MATERIALS AND METHODS}

\subsection{Experimental setup}

An ultrashort pulsed Ti:Sapphire laser (Coherent Chameleon Ultra II) was expanded 4 times to illuminate a liquid crystal on silicon spatial light modulator (LCoS-SLM, HSP1920 Meadowlark Optics). The laser delivers 140 -fs pulses with an $80-\mathrm{MHz}$ repetition rate, up to 4 -W average power with tunable central wavelength between 680 and $1080 \mathrm{~nm}$. The central wavelength was set to $800 \mathrm{~nm}$ and the laser power fed into the system was controlled via a half-wave plate and a polarizing beam-splitting cube. The first diffraction order was filtered out by an iris placed at the Fourier plane of a telescope that imaged the active area of the SLM onto a diffraction grating (600 lines/mm, Thorlabs). The spectrally dispersed beam was collimated with a $400 \mathrm{~mm}$ lens and relayed to the back focal plane of the illumination objective. An air objective $(20 \times$ numerical aperture [NA] $=0.75$; Nikon) was used to obtain a field-of-view (FOV) of approximately $90 \mu \mathrm{m} \times 90 \mu \mathrm{m}$. Fluorescent light was then collected via the same illumination objective in an epifluorescence geometry and detected either with an electron-multiplying charge-coupled device (EMCCD) camera (iXon ${ }^{\mathrm{EM}}+885$, Andor) like in our previous work ${ }^{26}$ or with a photomultiplier tube (PMT) (PMT2102, Thorlabs) digitized by a fast oscilloscope (PicoScope 5000 Series, pico Technology). Appropriate short-pass filters were used to reject illumination light. Samples, objective and detectors were mounted on the body of an inverted microscope (Eclipse Ti, Nikon) accordingly. The microscope was operated with software developed in MATLAB (r2018b).

To operate the microscope in the $3 \mathrm{P}$ mode the illumination wavelength was set to $1000 \mathrm{~nm}$ and the diffraction grating was replaced with a 300 lines/mm (Thorlabs) to obtain a narrower spectral dispersion. The objective was replaced with a $40 \mathrm{x}$ lens $(\mathrm{NA}=0.95$, Nikon) obtaining a FOV of $20 \mu \mathrm{m} \times 20 \mu \mathrm{m}$.

\subsection{Multiphoton excitation characterization}

It is well known that $2 \mathrm{P} / 3 \mathrm{P}$ fluorescence scales with the second/third power of the illumination intensity. ${ }^{28}$ The presence of multiphoton excitation signal in the blue fluorescent microparticles was tested by measuring the fluorescence intensity as the laser power was modulated. A laser beam (Coherent Chameleon Ultra II) was focused onto the sample and the emitted fluorescent light was detected with a CCD camera (Clara, Andor). Power control was achieved via a half-wave plate mounted on a motorized rotation stage paired with a polarizing beamsplitting cube. Different wavelengths were tested and the strongest and the most stable signals were observed at $750 \mathrm{~nm}$ for $2 \mathrm{P}$ excitation and at $1000 \mathrm{~nm}$ for $3 \mathrm{P}$ excitation. The measured values of the exponent were $2.00 \pm 0.01$ and $2.96 \pm 0.08$ for $2 \mathrm{P}$ and $3 \mathrm{P}$ excitation, respectively. In addition, the emission of PUREBLU 
Hoechst 33342 dye was characterized to demonstrate that 3P excitation can be achieved in biological specimens, obtaining a power dependence with exponent $3.16 \pm 0.03$.

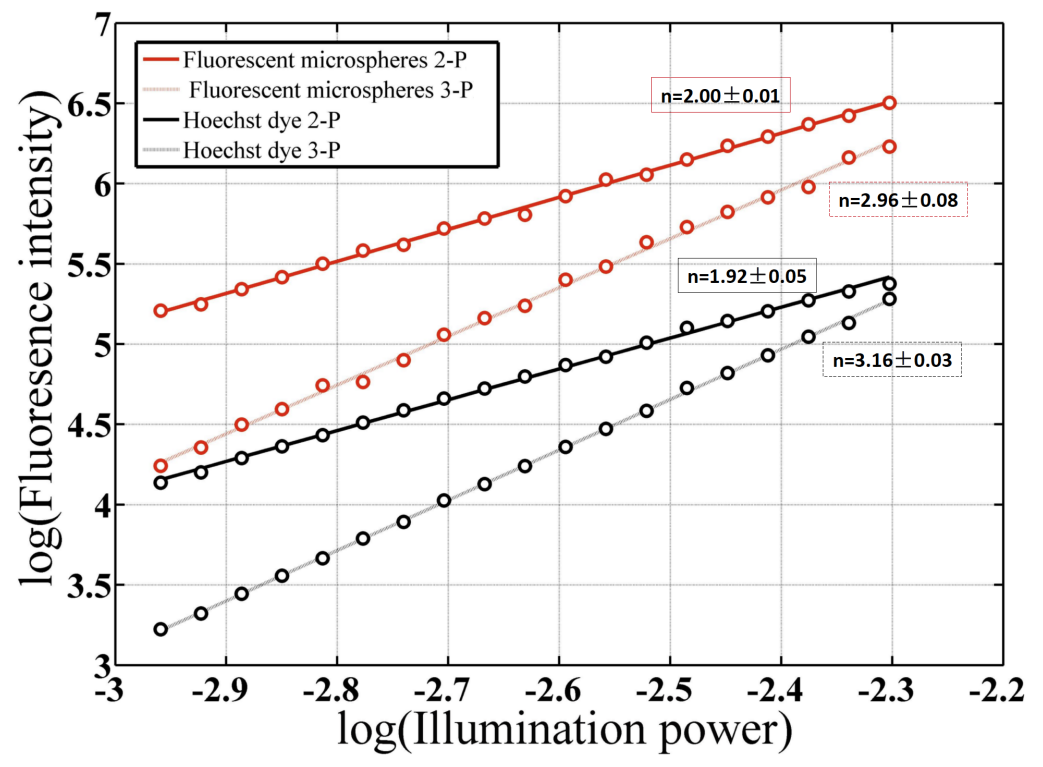

Figure 1. Quadratic and cubic fluorescence intensity dependence on laser power for blue fluorescent microspheres and Hoechst 33342 dye. A laser beam was focused on either of the samples and the fluorescence intensity was measured as the laser power was increased at constant linear steps starting from $50 \mathrm{~mW}$ to $100 \mathrm{~mW}$. Solid and dotted lines correspond to illumination at $700 \mathrm{~nm}(2 \mathrm{P})$ and $1000 \mathrm{~nm}(3 \mathrm{P})$, respectively. The gradient of the data displayed in logarithmic scale corresponds to the fluorescence intensity dependence on illumination power.

\subsection{Scattering phantom}

Polystyrene beads of $1 \mu \mathrm{m}$ in diameter (Microbead NIST Traceable Particle Size Standard, $1.00 \mu \mathrm{m}$; Polysciences) were mixed with a preheated $1 \%$ solution of agarose to simulate a uniformly scattering medium. The scattering phantom was designed to have scattering properties similar to biological tissue $\left(l_{s} \approx 140 \mu \mathrm{m}\right)$ similarly to our previous work. ${ }^{26}$ The solution was placed in wells made of multiple $90-\mu \mathrm{m}$ vinyl spacers attached on a glass coverslip.

\subsection{Fluorescent microspheres}

Green fluorescent polymer microspheres (G0500, $4.8 \mu \mathrm{m}$, Thermo Fisher Scientific) were embedded in a scattering phantom to test the imaging capability of the microscope in $2 \mathrm{P}$ mode in a volumetric scattering sample. Blue fluorescent polymer microspheres (B0200, $2.1 \mu \mathrm{m}$, Thermo Fisher Scientific) were used to study the performance of the system under 3P excitation. A small amount of blue microspheres solution was deposited on a glass coverslip and evaporated to generate a random distribution of beads.

\section{RESULTS}

\subsection{Principle of TRAFIX}

An expanded ultrashort pulsed laser beam illuminates an SLM that generates spatial patterns, for instance, in a Hadamard basis. The patterns are projected onto a diffraction grating that introduces geometrical dispersion and spreads out the spectral components of the beam. As a result, the pulse stretches in time. ${ }^{11,12}$ As the grating is re-imaged onto the sample, the initially introduced chirp is compensated by the objective lens, achieving temporal focusing. The initial ultrashort pulse duration is only recovered at the focal plane of the microscope objective, generating patterned axially confined multiphoton excitation. The use of temporal focusing ensures a nearly speckle-free propagation of the beam through scattering media enabling the projection of patterns onto 
the structure of interest. ${ }^{14,26}$ Fluorescent light emitted under each illumination pattern is collected via the same illumination objective in an epifluorescence geometry and is sensed with a single-element photo-detector. The single-pixel detector removes the need of resolving any spatial information in the detection system, which makes the microscope insensitive to light scattering in the detection system. Spatial resolution is obtained by using patterned illumination.

For a lossless single-pixel imaging measurement, one orthonormal pattern has to be projected for each pixel in the reconstructed image. Hence, a $n \times n$ pixel image needs $n^{2}$ patterns. For example, an image taken with a full set of 1024 patterns is composed of $32 \times 32$ pixels, while an image taken with a full Hadamard basis of 256 patterns contains only $16 \times 16$ in the same FOV. However, if the signal from the specimen is sparse in, for instance, the spatial frequency domain, the use of all patterns is not required to reconstruct a faithful representation of the original image. In fact, substantially fewer patterns can be used as long as they are mutually incoherent in the spatial frequency basis. This approach is usually termed compressive sensing. ${ }^{24,25,27}$

\subsection{Two-photon TRAFIX with compressive sensing}

We have recently demonstrated TRAFIX using $2 \mathrm{P}$ excitation, ${ }^{26}$ showing promise for deep imaging without correction. However, to achieve a broad utility in biomedicine, substantial consideration has to be placed in the efficient acquisition and recovery of the single-pixel signal. Compressive sensing and recovery can be used to significantly reduce the total number of patterns that are required and, thus, dramatically reduce imaging time. We demonstrate current advances in compressive sensing in simulation based on experimental data.

Figure 2(a) shows fluorescent microspheres imaged through a scattering phantom at a depth of approximately $300 \mu \mathrm{m}$ and recovered with no compression loss in the Hadamard basis, which we label as ground truth. Using only $5 \%$ and $15 \%$ of the least-sparse Hadamard vectors (A) and their respective single-pixel values from experiment $(y)$, we can reconstruct an approximation of the original image my minimizing the signal vector $\left(x=\mathrm{A}^{-1} y\right)$ to the $l_{1}$ norm in the spatial frequency domain. ${ }^{24,25,27}$ The results in Fig. 2(a) show that comparable recovery can be achieved with $15 \%$ of the recorded data, and the position of the beads, with some noise, can be recovered from $5 \%$ of the data. Here, for a 64 pixel-wide image, 205 and 615 patterns were used in recovery for $5 \%$ and $15 \%$ compression, respectively, which can be done in $<1 \mathrm{~s}$ and $<8$ GBs of memory. However, scaling this problem to higher sampled images is challenging as the recovery time and computational costs scale by more than the 4 -th power with image width. For instance, similar recovery of a $512 \times 512$ image would require in excess of 32 TBs of memory alone.

This problem has been at the forefront of compressive sensing. To form a discernible image, it is unnecessary to find the perfect most-sparse $x$; it is sufficient to find an approximation to $x$ with an acceptable error. As such, substantially more efficient methods involve making first-order simplifications to the reconstruction, and making use of a priori knowledge, for instance, that the image is smooth in the spatial domain. ${ }^{29}$ Furthermore, since we cannot know the least-sparse Hadamard vectors a priori for each sample, alternate sampling bases that are mutually incoherent are preferred; for instance, patterns that are generated from random Bernoulli trials. Figure $2(\mathrm{~b})$ shows an up-scaled image of the beads to $512 \times 512$ pixels. We sample the image with random Bernoulli patterns at $5 \%$ compression, such that approximately $13 \mathrm{k}$ patterns are used. The images are reconstructed by minimizing $x$ to an $l_{1}$ norm and total variation (TV), respectively, whilst being subject to a spatial smoothness quantified via an $l_{2}$ norm. ${ }^{29}$ Figure $2(\mathrm{~b})$ shows $512 \times 512$ reconstruction using the $l_{1}$ norm and $\mathrm{TV}$, taking $<3 \mathrm{~s}$ and $<1$ min, respectively. Experimentally, using our set-up, 13k patterns can be acquired in under 3.5 minutes. These results demonstrate that TRAFIX has the potential to reach the imaging performance of conventional microscopy, however, now at greater depths and lower photodamage.

\subsection{Three-photon TRAFIX}

Similarly to other multiphoton imaging techniques, TRAFIX can be extended to higher order multiphoton processes, such as $3 \mathrm{P}$ excitation, to potentially achieve better penetration depth. ${ }^{23,26} 3 \mathrm{P}$ microscopy usually relies on long wavelengths $(1300 \mathrm{~nm}$ and $1700 \mathrm{~nm})$, which are more resistant to scattering and achieve deeper penetration inside biological tissue. It has also been shown that by relying on such higher order nonlinear process, fluorescence is confined to a smaller volume, reducing out-of-focus light. ${ }^{17,22}$ Novel laser sources have recently been developed to generate high energy pulses to optimize the absorption of three photons. Such ultrashort 
(a)

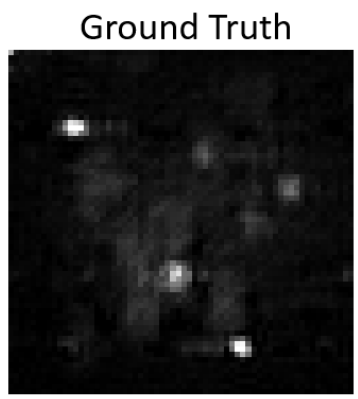

$64 \times 64$ pixels

(b)

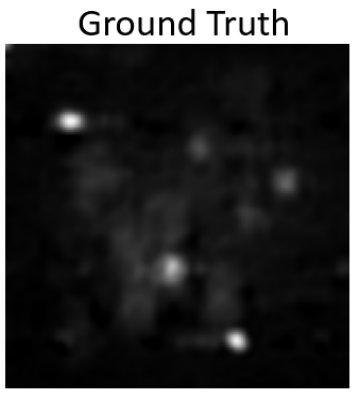

$512 \times 512$ pixels

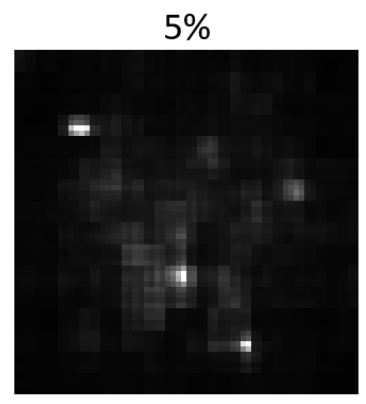

$$
\min \|x\|_{1}
$$



$\min \|x\|_{1}$

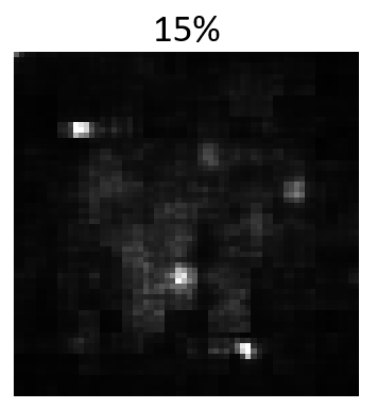

$\min \|x\|_{1}$

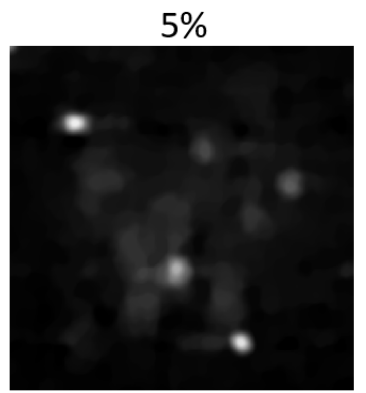

$\min \|x\|_{\mathrm{TV}}$

Figure 2. Two-photon TRAFIX. Images of $4.8 \mu \mathrm{m}$ green fluorescent microspheres embedded in a scattering phantom at a depth of approximately $300 \mu \mathrm{m}$. (a) Sample reconstructed with the full Hadamard basis sampled over $64 \times 64$ pixels. Compressive sensing is demonstrated a posteriori, by using $5 \%$ and $15 \%$ of the recorded Hadamard patterns and minimizing to the $l_{1}$ norm. (b) Simulated reconstruction of a $512 \times 512$ pixel sampled image using random Bernoulli patterns and $5 \%$ compression. Reconstruction is performed by minimizing to $l_{1}$ norm and TV, respectively, subject to a spatial smoothness $\left(l_{2}\right.$ norm) .

pulsed lasers typically have pulse duration and repetition rate below 70 fs and $1.25 \mathrm{MHz},{ }^{17-21,23}$ respectively. The Ti:Sapphire laser source in our system is typically used to generate $2 \mathrm{P}$ excitation, therefore, it is not optimal for $3 \mathrm{P}$ microscopy. Its long pulse duration (140 fs) and high repetition rate $(80 \mathrm{MHz})$ result in lower pulse energy that makes 3P imaging very inefficient. However, conventional Ti:Sapphire lasers can still be used to excite fluorophores at the high frequency end of the visible spectrum. ${ }^{22}$

To demonstrate that TRAFIX can potentially be used in 3P excitation mode, we imaged 2.1 um blue fluorescent microspheres. To obtain a high power per unit area and achieve enough photon density to generate $3 \mathrm{P}$ excitation, the FOV was reduced to only $20 \mu \mathrm{m} \times 20 \mu \mathrm{m}$. A small FOV results in a high illumination intensity but also in very fine details in the projected patterns. This becomes a problem when using large Hadamard bases since the patterns containing high frequencies cannot be projected onto the sample due to the diffraction limit. Therefore, the size of the Hadamard basis, i.e. the maximum number of Hadamard patterns used for imaging, has to be determined according to the NA and pupil size of the objective. In the present experiment, the microscope is designed to have a small FOV and, as a result, only $16 \times 16$ pixel patterns, i.e. 256 patterns, can be effectively projected. This results in strong pixelation in the images of beads under $3 \mathrm{P}$ excitation shown in Fig. 3 (c). Due to the limited pixel count in the image, individual beads cannot be resolved. However, the overall structure of the sample shown in the reference images (Fig.3 (a,b)) can be clearly identified in the TRAFIX images (Fig.3 (c,d)).

To increase the resolution, while maintaining a small FOV and the same spatial frequency content in the projected patterns, we implemented digital microscanning. ${ }^{30}$ In digital microscanning, low-resolution patterns 
$(n \times n)$ are laterally shifted by a sub-pixel amount and merged on a higher resolution grid. Overlapped regions are averaged and the resulting image is equivalent to a $2 n \times 2 n$ pixel image convolved with a smoothing kernel. Fig. 3 (c) shows four $16 \times 16$ pixel images obtained by shifting the projected light patterns and Fig. 3 (d) shows the $32 \times 32$ pixel image resulting after combination of the four previous images.

Reference

(a)

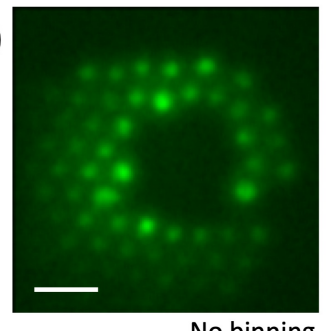

(b)

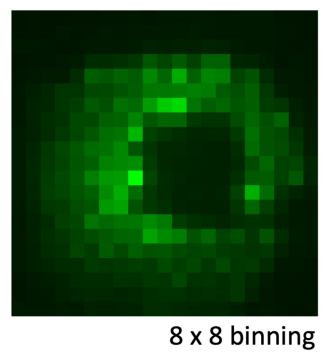

TRAFIX
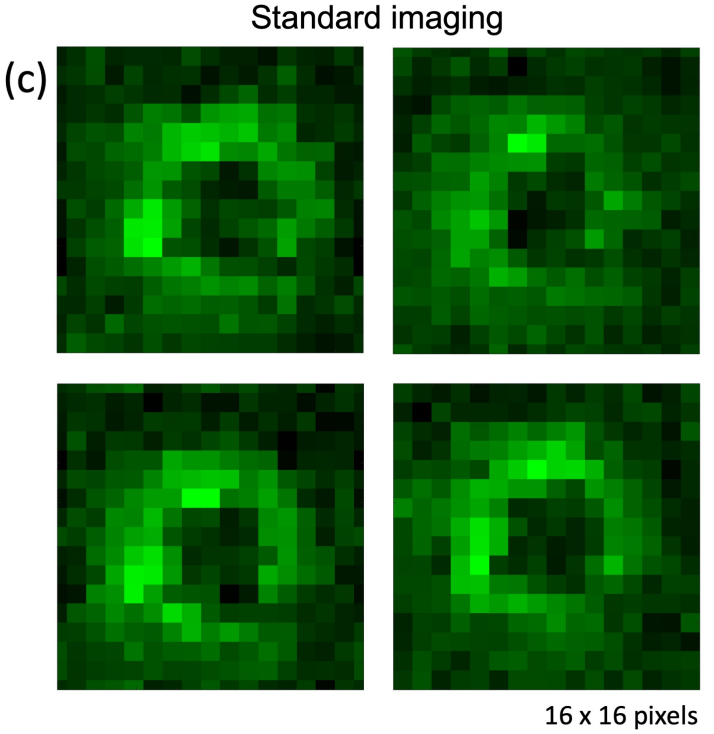

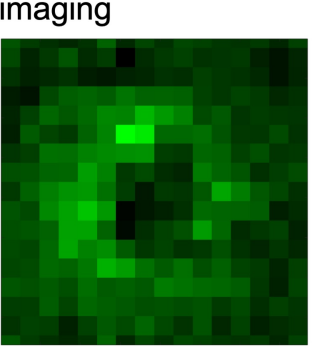

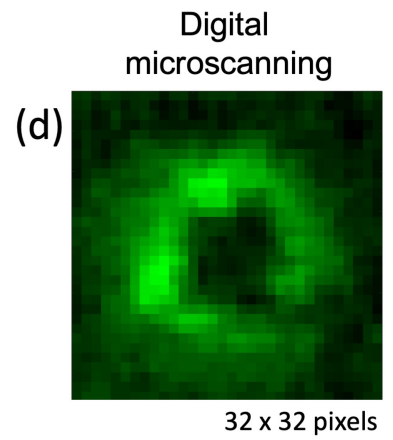

Figure 3. Preliminary data for three-photon excitation TRAFIX. Images of $2.1 \mu \mathrm{m}$ blue fluorescent microspheres imaged at $1000 \mathrm{~nm}$ wavelength. (a), (b) Reference images taken with an EMCCD camera under uniform temporal focusing illumination with (a) no pixel binning and with (b) $8 \times 8$ pixel binning. (c), (d) TRAFIX images without any scattering medium. (c) Low-resolution images $(16 \times 16$ pixel) obtained by shifting the 256 illumination Hadamard patterns by a suppixel amount. (d) High-resolution image reconstructed from the combination of the four digitally microscanned images in (c). Scale bar is $5 \mu \mathrm{m}$. False color.

\section{DISCUSSION AND CONCLUSIONS}

TRAFIX is a novel multiphoton microscope that combines temporal focusing with single-pixel detection to achieve wide-field imaging without correction at depth. In this work we have shown that TRAFIX can potentially be extended to the 3P excitation mode, and we have evaluated its prospects for compressive sensing.

Imaging through scattering media in $3 \mathrm{P}$ mode was not shown in this work due to the low $3 \mathrm{P}$ excitation efficiency using the current Ti:Sapphire laser. However, with the laser sources typically used in 3P microscopy, deeper imaging than in 2P TRAFIX will undoubtedly be achieved. Future studies will focus on the use of TRAFIX at the typical wavelengths for 3P excitation. By using the optimal laser for 3P TRAFIX, it will be possible to image large FOVs at high resolution. Nevertheless, even in the $3 \mathrm{P}$ excitation regime with the optimal laser source, it is expected that imaging depth will still be limited by the retention of the uniformity of the illumination patterns. Therefore, the optimal projection of patterns into depth should be investigated to achieve several millimeter deep imaging. Two-dimensional dispersion and pulse or wavefront shaping are two possibilities to be explored. Further research should also focus on the development of new illumination patterns that carry high spatial frequencies through turbid media more efficiently than the current Hadamard patterns.

In the first embodiment of TRAFIX, imaging speed was an important concern due to the use of a slow SLM and a highly binned EMCCD camera. In the current implementation presented here, imaging speed is increased to 60 frames-per-second thanks to the use of a faster SLM and a PMT. A complete imaging experiment with 
1024 patterns can now be performed in $17 \mathrm{~s}$. In addition, with the use of a state-of-the art DMD and compressive sensing, imaging speed could be vastly increased. ${ }^{31}$ Sub-1 min imaging and recovery is feasible for image sampling and FOVs matching conventional microscopy.

The intrinsic properties of TRAFIX (low photodamage, high penetration depth and high signal-to-background ratio), combined with 3P excitation and the state-of-the-art spatial light modulators, are likely to allow it to gain traction in biomedical sciences, such as neuroscience or cancer research. Compressive sensing, in particular, reduces the fundamental limit to information with which images can still be recovered. As such, imaging time and photodamage will always be prospectively lower. Therefore, further effort should be placed to refine compressive sensing methods in the context of microscopy, for instance, in propagating patterns effectively through scattering media.

\section{ACKNOWLEDGMENTS}

We thank James Glackin and Pavlos Manousiadis from University of St Andrews for support in the preparation of the uniform thin fluorescent layers of super yellow light-emitting PPV copolymer. We also thank Pengfei Liu for early stage work on sample characterization and Federico Gasparoli and Jonathan Nylk for useful discussions and support.

Funding: This work is supported by the UK Engineering and Physical Sciences Research Council for funding through grants EP/P030017/1 and EP/M000869/1, and has received funding from the European Union's Horizon 2020 Programme through the project Advanced BiomEdical OPTICAL Imaging and Data Analysis (BE-OPTICAL) under grant agreement no. 675512.

\section{REFERENCES}

[1] Betzig, E., Patterson, G. H., Sougrat, R., Lindwasser, O. W., Olenych, S., Bonifacino, J. S., Davidson, M. W., Lippincott-Schwartz, J., and Hess, H. F., "Imaging Intracellular Fluorescent Proteins at Nanometer Resolution," Science 313, 1642-1645 (2006).

[2] Hell, S. W. and Wichmann, J., "Breaking the diffraction resolution limit by stimulated emission: stimulatedemission-depletion fluorescence microscopy," Opt. Lett. 19, 780-782 (1994).

[3] Gustafsson, M. G., Shao, L., Carlton, P. M., Wang, C. J., Golubovskaya, I. N., Cande, W. Z., Agard, D. A., and Sedat, J. W., "Three-dimensional resolution doubling in wide-field fluorescence microscopy by structured illumination," Biophys. J. 94, 4957-4970 (2008).

[4] Power, R. M. and Huisken, J., "A guide to light-sheet fluorescence microscopy for multiscale imaging," Nat. Methods 14, 360-373 (2017).

[5] Booth, M. J., "Adaptive optical microscopy: the ongoing quest for a perfect image," Light Sci. Appl. 3, e165 (2014).

[6] Popoff, S. M., Lerosey, G., Carminati, R., Fink, M., Boccara, A. C., and Gigan, S., "Measuring the transmission matrix in optics: An approach to the study and control of light propagation in disordered media," Phys. Rev. Lett. 104, 100601 (2010).

[7] Mosk, A. P., Lagendijk, A., Lerosey, G., and Fink, M., "Controlling waves in space and time for imaging and focusing in complex media," Nat. Photonics 6, 283-292 (2012).

[8] Denk, W., Strickler, J. H., and Webb, W. W., "Two-photon laser scanning fluorescence microscopy," Science 248, 73-76 (1990).

[9] Katona, G., Szalay, G., Maák, P., Kaszás, A., Veress, M., Hillier, D., Chiovini, B., Vizi, E. S., Roska, B., and Rózsa, B., "Fast two-photon in vivo imaging with three-dimensional random-access scanning in large tissue volumes," Nat. Methods 9, 201-208 (2012).

[10] Katz, O., Small, E., Bromberg, Y., and Silberberg, Y., "Focusing and compression of ultrashort pulses through scattering media," Nat. Photonics 5, 372-377 (2011).

[11] Oron, D., Tal, E., and Silberberg, Y., "Scanningless depth-resolved microscopy," Opt. Express 13, 1468$1475(2005)$.

[12] Zhu, G., van Howe, J., Durst, M., Zipfel, W., and Xu, C., "Simultaneous spatial and temporal focusing of femtosecond pulses," Opt. Express 13, 2153-2159 (2005). 
[13] Schrödel, T., Prevedel, R., Aumayr, K., Zimmer, M., and Vaziri, A., "Brain-wide 3D imaging of neuronal activity in Caenorhabditis elegans with sculpted light," Nat. Methods 10, 1013-1020 (2013).

[14] Papagiakoumou, E., Bègue, A., Leshem, B., Schwartz, O., Stell, B. M., Bradley, J., Oron, D., and Emiliani, V., "Functional patterned multiphoton excitation deep inside scattering tissue," Nat. Photonics 7, 274-278 (2013).

[15] Pégard, N. C., Mardinly, A. R., Oldenburg, I. A., Sridharan, S., Waller, L., and Adesnik, H., "Threedimensional scanless holographic optogenetics with temporal focusing (3D-SHOT)," Nat. Commun. 8, 1228 (2017).

[16] Accanto, N., Molinier, C., Tanese, D., Ronzitti, E., Newman, Z. L., Wyart, C., Isacoff, E., Papagiakoumou, E., and Emiliani, V., "Multiplexed temporally focused light shaping for high-resolution multi-cell targeting," Optica 5, 1478 - 1491 (2018).

[17] Horton, N. G., Wang, K., Kobat, D., Clark, C. G., Wise, F. W., Schaffer, C. B., and Xu, C., "In vivo three-photon microscopy of subcortical structures within an intact mouse brain," Nat. Photonics 7, 205-209 (2013).

[18] Ouzounov, D. G., Wang, T., Wang, M., Feng, D. D., Horton, N. G., Cruz-Hernández, J. C., Cheng, Y. T., Reimer, J., Tolias, A. S., Nishimura, N., and Xu, C., "In vivo three-photon imaging of activity of GcamP6labeled neurons deep in intact mouse brain," Nat. Methods 14, 388-390 (2017).

[19] Guesmi, K., Abdeladim, L., Tozer, S., Mahou, P., Kumamoto, T., Jurkus, K., Rigaud, P., Loulier, K., Dray, N., Georges, P., Hanna, M., Livet, J., Supatto, W., Beaurepaire, E., and Druon, F., "Dual-color deep-tissue three-photon microscopy with a multiband infrared laser," Light Sci. Appl. 7, 2047-7538 (2018).

[20] Rodríguez, C., Liang, Y., Lu, R., and Ji, N., "Three-photon fluorescence microscopy with an axially elongated Bessel focus," Opt. Lett. 43, 1914 - 1917 (2018).

[21] Chen, B., Huang, X., Gou, D., Zeng, J., Chen, G., Pang, M., Hu, Y., Zhao, Z., Zhang, Y., Zhou, Z., Wu, H., Cheng, H., Zhang, Z., Xu, C., Li, Y., Chen, L., and Wang, A., "Rapid volumetric imaging with Bessel-Beam three-photon microscopy," Biomed. Opt. Express 9, 1992 - 2000 (2018).

[22] Escobet-Montalbán, A., Gasparoli, F. M., Nylk, J., Liu, P., Yang, Z., and Dholakia, K., "Three-photon light-sheet fluorescence microscopy," Opt. Lett. 43, 5484-5487 (2018).

[23] Rowlands, C. J., Park, D., Bruns, O. T., Piatkevich, K. D., Fukumura, D., Jain, R. K., Bawendi, M. G., Boyden, E. S., and So, P. T., "Wide-field three-photon excitation in biological samples," Light Sci. Appl. 6, e16255 (2017).

[24] Edgar, M. P., Gibson, G. M., and Padgett, M. J., "Principles and prospects for single-pixel imaging," Nat. Photonics 13 (2019).

[25] Durán, V., Soldevila, F., Irles, E., Clemente, P., Tajahuerce, E., Andrés, P., and Lancis, J., "Compressive imaging in scattering media," Opt. Express 23, 14424-14433 (2015).

[26] Escobet-Montalbán, A., Spesyvtsev, R., Chen, M., Saber, W. A., Andrews, M., Simon Herrington, C., Mazilu, M., and Dholakia, K., "Wide-field multiphoton imaging through scattering media without correction," Sci. Adv. 4, eaau1338 (2018).

[27] Candès, E. and Wakin, M., "An Introduction To Compressive Sampling," IEEE Signal Process. Mag. 25, 21-30 (2008).

[28] Xu, C., Zipfel, W., Shear, J. B., Williams, R. M., and Webb, W. W., "Multiphoton fluorescence excitation: new spectral windows for biological nonlinear microscopy.," Proc. Natl. Acad. Sci. U.S.A. 93, 10763-10768 (1996).

[29] Becker, S., Bobin, J., and Candès, E., "NESTA: A Fast and Accurate First-Order Method for Sparse Recovery," SIAM J. Imaging Sci. 4, 1-39 (2011).

[30] Sun, M.-J., Edgar, M. P., Phillips, D. B., Gibson, G. M., and Padgett, M. J., "Improving the signal-to-noise ratio of single-pixel imaging using digital microscanning," Opt. Express 24, 10476-10485 (2016).

[31] Alemohammad, M., Shin, J., Tran, D. N., Stroud, J. R., Chin, S. P., Tran, T. D., and Foster, M. A., "Widefield compressive multiphoton microscopy," Opt. Lett. 43, 2989 - 2992 (2018). 